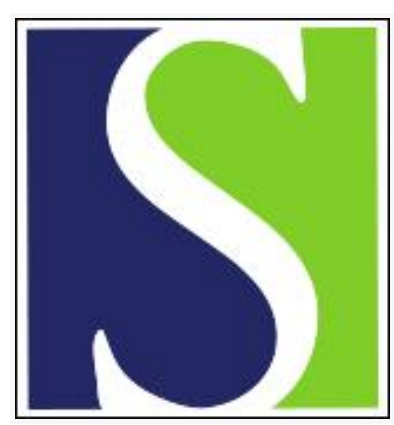

Scand J Work Environ Health 1987;13(4):313-316

https://doi.org/10.5271/sjweh.2046

Issue date: Aug 1987

Pathophysiological aspects of peripheral circulatory disorders in the vibration syndrome.

by Pyykko I, Gemne G

Affiliation: Institute of Occupational Health, Helsinki, Finland.

This article in PubMed: www.ncbi.nlm.nih.gov/pubmed/3324312 


\title{
Pathophysiological aspects of peripheral circulatory disorders in the vibration syndrome
}

\author{
by Ilmari Pyykkö, MD, PhD, ${ }^{1}$ Gösta Gemne, MD, PhD ${ }^{2}$
}

\begin{abstract}
PYYKKÖ I, GEMNE G. Pathophysiological aspects of peripheral circulatory disorders in the vibration syndrome. Scand J Work Environ Health 13 (1987) 313-316. A review of current knowledge on the pathophysiological aspects of peripheral circulatory derangements in the hand-arm vibration syndrome is presented. Hemodynamic measurements indicate that the primary factor in vibration-induced white finger is an increase in peripheral resistance of finger circulation, present after local and general cooling. The reason for this increase is not known, but it is postulated that there is an excess affinity for the efferent receptors of vasoactive substances and that this affinity is potentiated during local cooling of the digits.
\end{abstract}

Key terms: central vasomotor tone, finger circulation, peripheral resistance, receptor sensitivity.

\section{Etiological aspects of the vibration syndrome}

In attacks of vibration-induced white finger (VWF) the hyperreactivity of the blood vessels of subjects with VWF does not seem to be restricted to locally applied cold stimulus. Reflexively induced cold (4), noise (10), and vibratory stimuli (10) also cause an enhanced vasoconstriction in the affected vessels. It has therefore been proposed that excessive peripheral vasoconstriction may be produced by augmented vasoconstrictor nerve tone (10) or by faulty local factors $(6,7,11)$. In the case of the latter possibility the local vasoconstrictor mechanisms aggravate responses of the central output of the sympathetic nervous system (12). Such a situation generally occurs in outdoor workers when the body and the vessels of the fingers are cooled simultaneously.

Lewis (6) was the first to present the hypothesis that Raynaud's phenomenon is caused by local faults, ie, defects in nerve endings leading to excess vasoconstriction when the vessel wall is exposed to cold. Marshall et al (7) noticed that, in an attack of VWF, the numbness was more proximal in the fingers than the changes in color. They therefore proposed that in VWF, as in some neurological diseases, injured nerves were the primary reason for Raynaud's phenomenon. In an experiment on dogs Azuma et al (2) showed both in vivo and in vitro that a few hours after the termination of vibration the smooth muscles of the vessel wall responded more strongly to noradrenaline than unexposed muscles did.

Pyykkö et al (11) analyzed vasomotor oscillation in vibration-exposed subjects with and without finger

\footnotetext{
1 Institute of Occupational Health, Helsinki, Finland.

2 National Institute of Occupational Health, Stockholm, Sweden.
}

Reprint requests to: Dr I Pyykkö, Department of Otolaryngology, University Hospital of Helsinki, Haartmaninkatu 2-4 E, SF-00290 Helsinki, Finland. anesthesia. The neurogenic component of the vasomotor activity was found to be weaker in the VWF subjects than in referents. However, the authors found no evidence that the myogenic tone of vasomotor oscillation in VWF patients differed from that of healthy volunteers. The results therefore suggest that there is damage to the vasomotor nerves controlling the vessel tone, instead of damage in contracting proteins of the smooth muscle. Hence local neuropathy, as suggested by the results, may facilitate the "sprouting" of adrenergic receptors on the surface of the smooth muscles in the same manner as surgical sympathectomy produces hypersensitivity of the vessels.

Hypersensitivity of the vessel wall may cause much the same responses to exogenous noxious stimuli as does an enhanced central sympathetic outflow. Thus different sensory stimuli that have caused an excessive vasoconstriction reflexively in the finger circulation of vibration-exposed workers ultimately indicate a control lesion, but the fault may be peripheral (10). Moreover, because of the possible fault in the adrenergic receptors, the subjects with VWF show increased peripheral resistance to normal environmental stimuli and, respectively, show reduced finger skin temperatures and blood flow at normal ambient temperatures (4). In a cold environment the augmented vascular responses may lead to collapse of the vessel wall and thus provoke an attack of Raynaud's phenomenon.

Some other findings indicate that continuous stimulation of the pacinian corpuscles, which are very sensitive to vibration at frequencies of 60 to $700 \mathrm{~Hz}(10)$, might lead to continuous activation of the autonomic nervous system. Acute exposure to vibration also triggers a vasoconstriction reflexively in peripheral vessels. The peripheral vasoconstrictor response in the vessels is presumably caused by the activation of the autonomic nervous system, since, according to works on isolated animal postal vein, vibration affects smooth muscle tissue by causing vasodilatation. After prolonged stimulation such a repeated vasoconstriction 
may cause hypertrophy of the medial layer of the muscle wall as an exercise effect and lead to exaggerated vascular response to cold. Similar vascular hyperresponsiveness to noradrenaline, as in cases of VWF, has also been observed in hypertensive rats by Folkow et al (3) in cases in which the muscular layer of the arterioles, and of the arteriovenous shunts, was increased. Distant vibration may also cause changes in cardiovascular reflexes. Even though distant vibration may also induce central autonomic dysfunction, these effects of chronic exposure to vibration are still poorly documented.

So far the exact mechanism by which vibration induces a possible fault in the mechanisms controlling vessel tone remains to be elucidated. According to present knowledge it is likely that the fault in VWF might be in the coupling between the nerve and smooth mus-

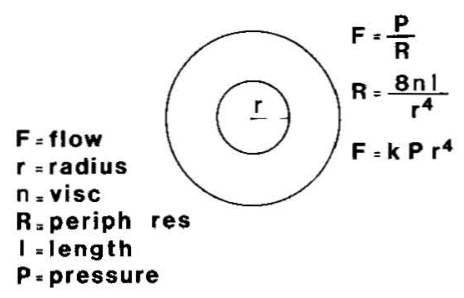

Figure 1. Relationship between pressure flow and peripheral resistance, as well as the determinants of peripheral resistance.
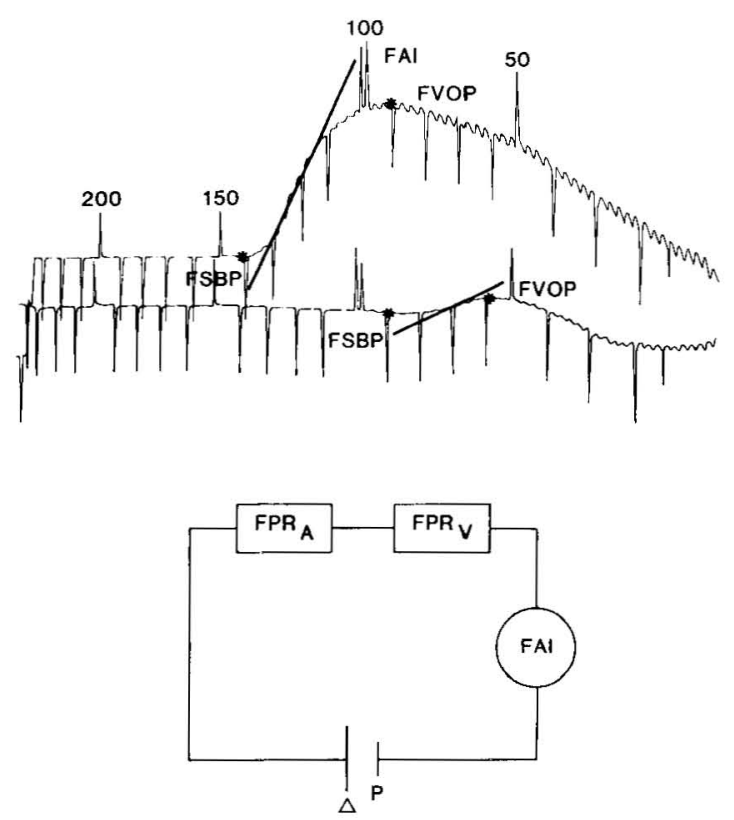

Figure 2. Upper diagram. Determination of finger arterial inflow (FAI), finger systolic biood pressure (FSBP), and finger venous opening pressure (FVOP) in uncooled and cooled finger from plethysmography tracings. Lower diagram. Evaluation of finger peripheral resistance. $\left(F P R_{A}=\right.$ arterial side of finger peripheral resistance, $F P R_{V}=$ venous side of finger peripheral resistance, total FPR $=$ a combination of $F P R_{A}$ and $F P R_{V}-$ Note that, when arterial inflow is measured, the pressure in the collecting cuff is decreasing the whole time, usually the arterial inflow is measured during one constant pressure). cle of the vessel at the adrenergic receptor - or, less likely, in the wall lumen ratio at the arteriolar level. Thus there is support for the theory that, in VWF, the fault may be local, in the finger vessels, but the nature and the exact mechanisms of the "excessive vasospasm" must be clarified before any final conclusions can be drawn.

\section{Hemodynamic aspects of vibration-induced white finger}

Hitherto, the importance of the different factors determining blood circulation in patients with VWF has not been understood in detail. There is a well known connection between pressure, flow, and peripheral resistance. Thus flow is inversely related to peripheral resistance and directly related to pressure. The peripheral resistance is dependent on an individual constant (k), the length of the capillary bed (l), the viscosity of the blood $(\mathrm{m})$, and the radius of the lumen of the vessel (r), as described in figure 1 .

Although local mechanisms operating on the vessel wall of the resistance arterioles and capillary beds may contribute to vasoconstriction (15), the outflow of the sympathetic vasoconstrictor nerves is far more important. This outflow is aimed at the resistance arterioles, precapillary sphincters, and arteriovenous anatomosis (11). According to Poisseuille's law a slight decrease in the radius causes a powerful increase in the peripheral resistance and results in a reduction in flow, provided that the pressure is not changed. Thus under physiological conditions, changes in the radius of the vessel lumen provide an efficient way of controlling skin circulation.

To evaluate possible changes in peripheral resistance, invasive measurements are at present more accurate. However, the technical difficulties and size of measuring devices at present do not allow the instruments to be placed in digital arteries and arterioles without severely occluding the vessel lumen. Furthermore, finger circulation can resist high venous pressure and still provide satisfactory flow, as during the manipulation of working tools with a relatively firm grip. Therefore, the difference in pressure acting as the driving force varies greatly in the finger, depending on the changes on the venous side. A monitoring of arterial pressure alone may thus provide biased results.

One possible way to approach the determination of the peripheral resistance of the system is to use finger plethysmography with the strain gauge technique (12). In this kind of approach the circulation is a net result of the combined flow pattern of the arterial and venous sites (figure 2) and can be measured as peak arterial inflow $(5,12)$. A crude estimation of the pressure difference, the so-called driving force, can be made from a calculation of the difference between finger systolic blood pressure and venous opening pressure. In this way the finger peripheral resistance that derives from 
the arterial and venous side can be estimated at different ambient temperatures. This procedure is somewhat different from the usual procedure in which mean arterial pressure and maximal arterial flow is used. Nevertheless, it is extremely difficult, though not impossible, to measure mean arterial pressure noninvasively.

Using this approach, Pyykkö (12) and Gemne et al (5) determined finger peripheral resistance during local cooling in subjects with and without VWF. The results showed, in fact, that in subjects with VWF the arterial inflow was limited as the result of high peripheral resistance that appeared after local cooling of the fingers. Locally applied cold thus triggered a higher peripheral resistance in the finger vessels of subjects with VWF than in the finger vessels of referents. Furthermore, this increase in peripheral resistance was only partly reduced after the finger nerves were anesthetized (blocking the sympathetic nervous control on the finger vessels) and therefore indicated that an augmentation of local vascular mechanisms is the immediate cause of VWF.

Local exposure to cold affects the alpha-adrenergic receptors and promotes the vasoconstriction induced by the sympathetic nervous system via two different mechanisms, ie, reducing the degradation of transmittor substances and sensitizing the affinity of alphaadrenergic receptors to transmittor substances (15). The slowing of the degradation of transmittor substances is achicved by the inhibition of neuronal uptake, by delayed enzymatic degradation, and by retarded diffusion of noradrenaline. Thus cooling the vessels is an effective way to "prime" an attack in subjects with VWF, in whom presumably the local vascular control mechanism is lesioned. This receptor "priming" effect of cold is allegedly the reason why cold provokes attacks of VWF.

Some studies have demonstrated that subjects with VWF have higher blood viscosity than those without VWF (8). Since blood viscosity also influences peripheral resistance, it seems likely that those subjects with high or pathological viscosity of blood tend to be selected to the group with VWF and that the condition may not be directly caused by hand-arm vibration.

Structural changes have also been found in workers exposed to vibration (14). Intimal thickening and hypertrophy of the medial layer are the most characteristic findings. Since these factors affect the lumen of the digital arteries and reduce the radius of the vessels, they increase peripheral resistance. Nevertheless, no physiological study has yet been able to show any distinct effects on blood circulation which could be explained by structural changes. Therefore, this factor may be a marginal one. However, from the hemodynamic point of view, cooling combined with compression of the skin of the fingers reduces the patency and the length of the finger vessels and results in an increase in peripheral resistance, which may secondarily lead to a reduction in blood flow in VWF. This mechanism may have functional significance in triggering attacks.

It has been suggested that exposure to vibration produces "friction" in the skin of the fingers and leads to the formation of subcutaneous callosities (13). The calloused finger pads cause capillary occlusion affecting a part of the capillary bed. This occurrence decreases the blood volume in the capillaries and hence weakens the buffer action to finger circulation during sudden contractions of the finger vessels and therefore leads to an attack of VWF. Substitution of subcutaneous tissue by callosities during prolonged vibration reduces the size of the capillary bed and the apparent length of the vascular bed and also augments peripheral resistance. The hemodynamic significance of this mechanism remains to be elucidated.

An increase in general sympathetic tone induced either by emotional tension or by drugs such as nicotine also favors the outbreak of symptoms (9). A part of the prominent increase in peripheral resistance in subjects with VWF undoubtedly occurs due to central sympathetic output (4). Nevertheless, a pathologically high peripheral resistance during cold has been shown to persist after blockage of the sympathetic nerves to the finger (5), a phenomenon indicating that local factors play a far more important role in the genesis of VWF than do central factors.

During local cooling an increase in venous tone has also been observed in subjects with VWF. The elevated venous tone may increase the venous pressure and retard circulation by reducing the dynamic range of circulation. It has been demonstrated that high venous pressure retards the arterial inflow through the venoarterial reflex. As a net result, changes in the venous side have a powerful effect on peripheral resistance.

\section{Conclusions}

Several vascular factors contribute to the generation of an attack of VWF. These factors all cause increased peripheral resistance. An enhancement of blood viscosity may operate in much the same manner as structural changes in the vessel wall and may be a "selecting" factor, rather than an etiologic factor, that favors the worker's becoming a victim of VWF.

An increase in venous tone appears to be an important hemodynamic response which, through the venoarterial reflex and the increased venous flow resistance, "primes" for gross vascular changes on the arterial side. Local cold efficiently affects the vasoconstriction by retarding a reuptake of noradrenaline and by sensitizing the alpha receptors. This occurrence explains why attacks are provoked by cold instead of by mental or emotional stress. The latter factors, however, elevate the central sympathetic outflow and presumably assist the local faulty mechanisms in provoking the attack. 


\section{References}

1. Abrahams DI. Circulation in the extremities. Academic Press Inc, New York, NY 1967, p 557.

2. Azuma T, Ohhashi T, Sakaguchi M. Vibration-induced hyperresponsiveness of arterial smooth muscle to noradrenaline with special reference to Raynayd's phenomenon in vibration disease. Cardiovasc Res 12 (1978) $758-764$.

3. Folkow B, Hallbäck M, Lundgren Y, Weiss L. Background of increased flow resistance and vascular reactivity in spontaneously hypertensive rats. Acta Physiol Scand 80 (1970) 93-106.

4. Futatsuka M, Pyykkö I, Färkkilä M, Korhonen O, Starck J. Blood pressure, flow and peripheral resistance of digital arteries in vibration syndrome. $\mathrm{Br} \mathrm{J}$ Ind Med 40 (1983) 434-441.

5. Gemne G, Pyykkö I, Starck J, Ilmarinen R. Circulatory reaction to heat and cold in vibration-induced white finger with and without sympathetic blockade - An experimental study. Scand J Work Environ Health 12 (1986) $371-377$.

6. Lewis T. Vascular disorders of the limbs. Second edition. MacMillan, London 1949.

7. Marshall J, Poole EB, Reynard WA. Raynaud's phenomenon due to vibration tools. Lancet 1 (1954) 1151-
1156.

8. Okada A, Yamashita T, Nagano C, Ikeda T, Yachi A, Shobata S. Studies on the diagnosis and pathogenesis of Raynaud's phenomenon of occupational origin. $\mathrm{Br}$ J Ind Med 28 (1971) 353-357.

9. Pyykkö l. The prevalence and symptoms of traumatic vasospastic disease among lumberjacks in Finland. Work Environ Health 11 (1974) 118-131.

10. Pyykkö I. A physiological study of the vasoconstrictor reflex in traumatic vasospastic disease. Work Environ Health 11 (1974) 170-186.

11. Pyykkö 1, Gemne G, Starck J, llmarinen R, Aalto H. Vasomotor oscillation in vibration-induced white finger. Scand J Work Environ Health 12 (1986) 389-394.

12. Pyykkö I. Finger peripheral resistance during local cooling in vasospastic disease. Scand J Work Environ Health 12 (1986) 395-399.

13. Steward AM, Goda DF. Vibration syndrome. Br J Ind Med 27 (1979) 19-27.

14. Takeuchi T, Imanishi J. Histopathological observations in finger biopsy from thirty patients with Raynaud's phenomenon of occupational origin. J Kumamoto Med Soc 58 (1984) 54-70.

15. Vanhoutte PM, Janssens WJ. Thermosensitivity of cutaneous vessels and Raynaud's disease. Am Heart J 100 (1980) 263-265. 